\title{
SCREENING OF INTERMOLECULAR FORCES IN ADSORBATES
}

\author{
J. MaHANTY \\ Department of Theoretical Physics, Research School of Physical Sciences \& Engineering \\ The Australian National University \\ Canberra, A.C.T. 2601, Australia \\ (Received May 21, 1991)
}

\begin{abstract}
The interaction potential of a pair of adsorbed molecules differ from that of the same molecules in free space, because of the response of the substrate. This response does not influence much the short-range repulsive part of the potential; only the long-range attractive part is affected. The change due to the influence of the substrate has been the subject of extensive study, both theoretically and experimentally. In this paper the problem is formulated in terms of the dynamic charge response properties of the interacting pair, and applications to the case of adsorbed molecules on model metal surfaces is described.
\end{abstract}

PACS numbers: 82.65.-i, 68.90.+g

\section{Introduction}

The nature of intermolecular forces in free space is well understood. The interaction potential of a pair of molecules as a function of the separation distance $R$ has a canonical form (Fig. 1), with a short-range repulsive part and a long-range attractive part. The former is due to the overlap of the electrons of the two molecules at close separations, which leads to an increase in the kinetic and Coulomb energies of the electrons. The attractive tail is essentially the London-van der Waals $(\mathrm{L}-\mathrm{vdW})$ or dispersion interaction between the molecules.

A commonly used expression to fit this intermolecular potential curve is the Lennard-Jones potential,

$$
V(R)=\frac{A}{R^{12}}-\frac{B}{R^{6}} .
$$

Here the second term is essentially the L-vdW potential and its strength is $B$.

When the two interacting molecules are imbedded in a medium, the repulsive part is not much affected, because this part occurs at close separations, where intervention by the medium is not significant. But the L-vdW interaction, which arises from the correlated polarization fluctuations of the two molecules, can 


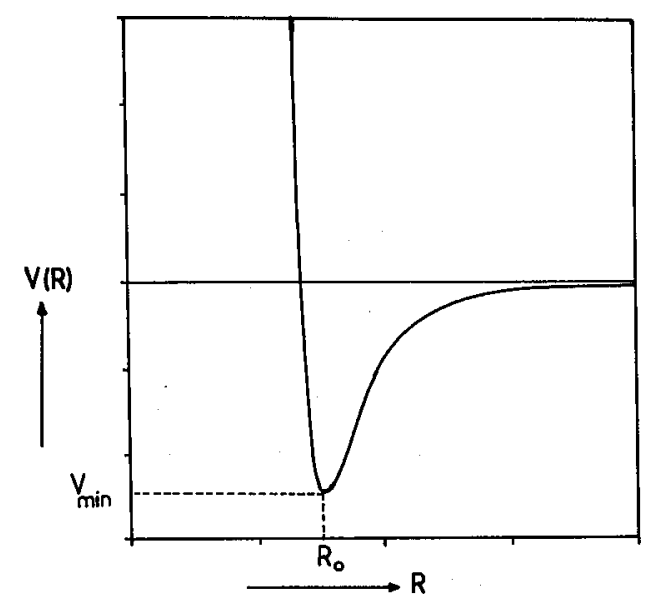

Fig. 1. The canonical form of the intermolecular interaction potential, with short-range repulsive and long-range attractive parts.

change substantially due to the response of the medium to the field associated with these polarization fluctuations. Thus, if we take the potential in the Lennard-Jones form, $B$ will depend on the environment. The minimum of the potential occurs at $R_{0}=(2 A / B)^{1 / 6}$, and $V_{\min }=-\left(B^{2} / 4 A\right)$. $R_{0}$ does not depend much on the value of $B$, but $V_{\min }$ depends rather sensitively on it. $B$ is generally reduced under the influence of the medium, so that $V(R)$ becomes shallower.

The proximity of a surface, such as would be the case with adsorbed molecules, causes a similar change in $\mathrm{L}-\mathrm{vdW}$ interaction of two molecules due to the response of the surface to their polarization fluctuations. This process is easily understood for physisorbed atoms or molecules, and in this paper we shall deal with an analysis of this aspect. It must be stressed that the effect of the surface on L-vdW interaction is only one of the factors affecting the lateral interaction of adsorbate molecules. Factors such as induced moments on adsorbed molecules will contribute to the long-range interaction between them, and the corrugation of the surface associated with the substrate atomic arrangement also has a role. We shall not deal here with these aspects, nor shall we deal with chemisorbed molecules. Chemisorbed molecules are not very mobile along the surface, and the concept of intermolecular interaction potential is not very well-defined for them.

The subject of adsorption being an old one, there is a vast literature covering experimental and theoretical work on the interaction of adsorbate molecules. Some of the recent reviews are by Bruch [1], March [2], and an earlier one by Takaishi [3]. The experimental evidence for change in intermolecular interaction between physisorbed molecules from its free space value is prolific, but not in all cases is the explanation simple. In one instance, that of Xe adsorbed on $W$, the strength of Xe-Xe interaction can be extracted from the data on desorption energy obtained by thermal desorption experiments, and the reduction in the strength has 
been explained by Joyce et al. [4] as due to the surface response to polarization fluctuations which is the subject of this paper. Much of the earlier data on the lateral interaction of adsorbates was obtained from thermal desorption experiments. Among the recent advances in experimental techniques, the most promising one is study of surfaces by IIe scattering [5], which has opened up possibilities of accurate determination of the pair distribution function of adlayers; this of course is directly related to the intermolecular interaction potential.

We now proceed to develop the theoretical framework for taking account of the substrate response, and its effect on intermolecular forces in adsorbates.

\section{Theoretical approaches}

Originally, the method given by London [6] for evaluation of L-vdW interaction of two molecules was to treat the Coulomb interaction of the charges in the two molecules as a perturbation, and the interaction potential is then the energy shift of the two molecules evaluated by perturbation theory. The effect of any environment would be to modify the Coulomb interaction of the charges. In the simplest form this can be obtained from the dielectric properties of the medium. It is advantageous to formulate the problem in a way that takes into account the dynamic charge response properties of the medium, so that the frequency dependence of the response of the medium is automatically included in the formalism. This will be done now.

For a pair of interacting atoms $A$ and $B$ with atomic numbers $Z_{A}$ and $Z_{B}$, the Hamiltonian

$$
H=H_{A}+H_{B}+V_{A B} \equiv H_{0}+V_{A B}
$$

Here $V_{A B}$ is the Coulomb interaction of the charges in $A$ and those in $B$.

Unlike in the original formulation of London [6], a proper perturbation theoretic evaluation of the interaction energy must take into account the exchange symmetry of the electrons in the two atoms. Forms of perturbation theory preserving exchange symmetry have been developed by Hirshfelder [7], Rumyantsev [8], and others. In the first order, using this approach, we get the leading terms in the Coulomb and exchange interactions, and in the second order, the L-vdW interaction with exchange corrections. The exchange corrections to $\mathrm{L}-\mathrm{vdW}$ interaction become insignificant for large separations, as may be expected.

The second order term in perturbation theory can be cast in a form that relates it to the dynamic charge response properties of the interacting atoms. For simplicity in establishing the relationship we start with ordinary perturbation theory without exchange corrections. We can then write $V_{A B}$ in the form,

$$
V_{A B}=\int \frac{\rho_{A}(r) \rho_{B}\left(r^{\prime}\right) \mathrm{d}^{3} r \mathrm{~d}^{3} r^{\prime}}{\left|r-r^{\prime}-R\right|}
$$

$\boldsymbol{R}$ being the distance of the centre of $B$ from the centre of $A$. The origin of $\boldsymbol{r}$ is the centre of $A$, and that of $r^{\prime}$ is the centre of $B$. The charge densities $\rho_{A}(r)$ and $\rho_{B}(r)$ are given by

$$
\rho_{A}(r)=-e \sum_{i}^{Z_{A}} \delta\left(r_{i}^{(A)}-r\right)+Z_{A} e \delta(r),
$$




$$
\rho_{B}(\boldsymbol{r})=-e \sum_{j}^{Z_{B}} \delta\left(r_{j}^{(B)}+R-r\right)+Z_{B} e \delta(r-R) .
$$

$r_{i}^{(A)}$ and $r_{j}^{(B)}$ are the electron coordinates in $A$ and $B$. The superscripted $r^{(A)}$ and $r^{(B)}$ will respectively be from the centres of $A$ and $B$.

The leading term in the interaction energy for spherically symmetric atoms is the second order shift due to $V_{A B}$, given by

$$
E(R)=-\sum_{n}^{\prime} \frac{\left|\left(V_{A B}\right)_{0, n}\right|^{2}}{\varepsilon_{n}-\varepsilon_{0}},
$$

with the symbols having their usual meaning.

Using the representation

$$
\begin{aligned}
& \frac{1}{\varepsilon_{n}-\varepsilon_{0}} \equiv \frac{1}{\left(\varepsilon_{n(A)}-\varepsilon_{0(A)}\right)+\left(\varepsilon_{n(B)}-\varepsilon_{0(B)}\right)} \\
& =\frac{2 \omega_{n(A)} \omega_{n(B)}}{\pi \hbar} \int_{0}^{\infty} \frac{\mathrm{d} \xi}{\left(\omega_{n(A)}^{2}+\xi^{2}\right)\left(\omega_{n(B)}^{2}+\xi^{2}\right)}
\end{aligned}
$$

with $n \equiv n(A)+n(B)$, and $\omega_{n(A, B)} \equiv\left[E_{n(A, B)}-E_{0(A, B)}\right] / \hbar$, and taking the product form of the wave function of the system,

$$
\psi_{n} \equiv \psi_{n(A)}\left(\left\{r_{i}(A)\right\}\right) \psi_{n(B)}\left(\left\{r_{j}(B)\right\}\right)
$$

Eq. (2.4) can be written as

$$
\begin{aligned}
& E(R)=-\frac{\hbar}{2 \pi} \frac{(2 \pi)^{12}}{4 \pi^{4}} \int_{0}^{\infty} \mathrm{d} \xi \int \frac{\mathrm{d}^{3} k_{1} \mathrm{~d}^{3} k_{2}}{k_{1}^{2} k_{2}^{2}} \Gamma_{A}\left(k_{1}, k_{2} ; \mathrm{i} \xi\right) \\
& \times \Gamma_{B}\left(-k_{1},-k_{2} ; \mathrm{i} \xi\right) \exp \left[\mathrm{i} R \cdot\left(k_{1}-k_{2}\right)\right] .
\end{aligned}
$$

Here

$$
\begin{aligned}
& \Gamma_{A}\left(k_{1}, k_{2} ; \omega\right)=\frac{1}{(2 \pi)^{6}} \int \chi_{A}\left(r_{1}^{(A)}, r_{2}^{(A)} ; \omega\right) \\
& \times \exp \left[-\mathrm{i}\left(k_{1} \cdot r_{1}^{(A)}-k_{2} \cdot r_{2}^{(A)}\right)\right] \mathrm{d}^{3} r_{1}^{(A)} \mathrm{d}^{3} r_{2}^{(A)},
\end{aligned}
$$

with

And

$$
\begin{aligned}
& \chi_{A}\left(r_{1}^{(A)}, r_{2}^{(A)} ; \omega\right) \equiv \sum_{n(A)}^{\prime} \frac{2 \omega_{n(A)}}{\hbar\left(\omega_{n(A)}^{2}-\omega^{2}\right)}\left[\rho_{A}\left(r_{1}^{(A)}\right)\right]_{O(A), n(A)} \\
& \times\left[\rho_{A}\left(r_{2}^{(A)}\right)\right]_{0(A), n(A)}
\end{aligned}
$$

$$
\begin{aligned}
& \Gamma_{B}\left(k_{1}, k_{2} ; \omega\right)=\frac{1}{(2 \pi)^{6}} \int \chi_{B}\left(r_{1}^{(B)}, r_{2}^{(B)} ; \omega\right) \\
& \times \exp \left[-\mathrm{i}\left(k_{1} \cdot r_{1}^{(B)}-k_{2} \cdot r_{2}^{(B)}\right)\right] \mathrm{d}^{3} r_{1}^{(B)} \mathrm{d}^{3} r_{2}^{(B)}
\end{aligned}
$$


with

$$
\begin{aligned}
& \chi_{B}\left(r_{1}^{(B)}, r_{2}^{(B)} ; \omega\right) \equiv \sum_{n(B)}^{\prime} \frac{2 \omega_{n(B)}}{\hbar\left(\omega_{n(B)}^{2}-\omega^{2}\right)}\left[\rho_{B}\left(r_{1}^{(B)}\right)\right]_{O(B), n(B)} \\
& \times\left[\rho_{B}\left(r_{2}^{(B)}\right)\right]_{O(B), n(B)}
\end{aligned}
$$

Here $r_{1,2}^{(A)}$ and $r_{1,2}^{(B)}$ are dummy variables, not to be confused with electron coordinates.

In terms of the Fourier transforms of the charge distributions

$$
\rho_{A, B}(k)=\frac{1}{(2 \pi)^{3}} \int \rho_{A, B}\left(r^{(A, B)}\right) \exp \left[-\mathrm{i} k \cdot r^{(A, B)}\right] \mathrm{d}^{3} r^{(A, B)},
$$

$\Gamma_{A}$ and $\Gamma_{B}$ can be written as

$$
\begin{aligned}
& \Gamma_{A}\left(k_{1}, k_{2} ; \omega\right) \equiv \sum_{n(A)}^{\prime} \frac{2 \omega_{n(A)}}{\hbar\left(\omega_{n(A)}^{2}-\omega^{2}\right)} \\
& \times\left[\rho_{A}\left(k_{1}\right)\right]_{0(A), n(A)}\left[\rho_{A}\left(-k_{2}\right)\right]_{n(A), 0(A)}, \\
& \Gamma_{B}\left(k_{1}, k_{2} ; \omega\right) \equiv \sum_{n(B)}^{\prime} \frac{2 \omega_{n(B)}}{\hbar\left(\omega_{n(B)}^{2}-\omega^{2}\right)} \\
& \times\left[\rho_{B}\left(k_{1}\right)\right]_{0(B), n(B)}\left[\rho_{B}\left(-k_{2}\right)\right]_{n(B), 0(B)},
\end{aligned}
$$

with

$$
\begin{aligned}
& \rho_{A}(k)=\frac{e}{(2 \pi)^{3}}\left[Z_{A}-\sum_{i}^{Z_{A}} \exp \left\{-\mathrm{i} k \cdot r_{i}^{(A)}\right\}\right] \\
& \rho_{B}(k)=\frac{e}{(2 \pi)^{3}}\left[Z_{B}-\sum_{j}^{Z_{B}} \exp \left\{-\mathrm{i} k \cdot r_{j}^{(B)}\right\}\right] .
\end{aligned}
$$

The functions $\chi_{A, B}\left(r_{1}^{(A, B)}, r_{2}^{(A, B)} ; \omega\right)$ are the charge response functions of the two atoms, in the sense that in the presence of an external potential $\varphi_{\text {ext }}(r, \omega)$ with frequency $\omega$, the induced charge density on the atoms is given by

$$
\begin{aligned}
& \rho_{\mathrm{I}(A, B)}\left(r^{(A, B)} ; \omega\right)=\int \chi_{A, B}\left(\boldsymbol{r}^{(A, B)},{\gamma^{\prime}}^{(A, B)} ; \omega\right) \\
& \times \varphi_{\operatorname{ext}}\left({r^{\prime}}^{(A, B)}, \omega\right) \mathrm{d}^{3} r^{(A, B)} .
\end{aligned}
$$

This equation provides a basis for evaluation of the interaction energy in terms of the response functions. If we start with an induced charge $\rho_{\mathrm{I}(B)}\left(r^{(B)}, \omega\right)$ on $B$, this will impose a potential on $A$, which in its turn will induce a charge on $A$. The potential at $B$ due to this charge on $A$ can be considered as the cause of the origin of $\rho_{\mathrm{I}(B)}$. We thus get a homogeneous integral equation for $\rho_{\mathrm{I}(B)}$ in the form

$$
\rho_{\mathrm{I}(B)}\left(r_{2}^{(B)}, \omega\right)=\int K_{B}\left(r_{2}^{(B)}, r_{1}^{(B)} ; \omega\right) \rho_{\mathrm{I}(B)}\left(r_{1}^{(B)}, \omega\right) \mathrm{d}^{3} r_{1}^{(B)}
$$


where the kernel $K_{B}$ has the explicit form

$$
\begin{aligned}
& K_{B}\left(r_{2}^{(B)}, r_{1}^{(B)} ; \omega\right) \\
& =\int \frac{\chi_{B}\left(r_{2}^{(B)}, r_{3}^{(B)} ; \omega\right) \chi_{A}\left(r_{2}^{(A)}, r_{1}^{(A)} ; \omega\right) \mathrm{d}^{3} r_{3}^{(B)} \mathrm{d}^{3} r_{2}^{(A)} \mathrm{d}^{3} r_{1}^{(A)}}{\left|r_{3}^{(B)}+R-r_{2}^{(A)}\right|\left|r_{1}^{(A)}-r_{1}^{(B)}-R\right|}
\end{aligned}
$$

Taking the Fourier transform of Eq. (2.14), and using Eqs. (2.15), (2.8) and (2.9), we get

$$
\begin{aligned}
& \rho_{\mathrm{I}(B)}(k)=\frac{(2 \pi)^{12}}{2 \pi^{4}} \int \frac{\mathrm{d}^{3} k_{1} \mathrm{~d}^{3} k^{\prime}}{k_{1}^{2} k^{\prime 2}} \Gamma_{B}\left(-k,-k_{1} ; \omega\right) \Gamma_{A}\left(k_{1}, k^{\prime} ; \omega\right) \\
& \times \exp \left[\mathrm{i} R \cdot\left(k_{1}-k^{\prime}\right)\right] \rho_{\mathrm{I}(B)}\left(k^{\prime}\right)=\frac{(2 \pi)^{18}}{4 \pi^{4} \Omega^{2}} \\
& \times \sum_{k_{1}, k^{\prime}} \frac{\Gamma_{B}\left(-k,-k_{1} ; \omega\right) \Gamma_{A}\left(k_{1}, k^{\prime} ; \omega\right) \exp \left[\mathrm{i} R \cdot\left(k_{1}-k^{\prime}\right)\right]}{k_{1}^{2} k^{\prime 2}} \rho_{\mathrm{I}(B)}\left(k^{\prime}\right) .
\end{aligned}
$$

where $\Omega$ is the normalization volume, and the integration over $k$ is replaced by a summation using $\int \mathrm{d}^{3} k \equiv\left[8 \pi^{3} / \Omega\right] \sum_{k}$.

The solvability condition of Eq. (2.16) is the vanishing of the determinant

$$
|\mathcal{I}-\mathcal{S}(\omega)|=0,
$$

where the elements of the matrix $\mathcal{S}(\omega)$ are given by

$$
\begin{aligned}
& S\left(k, k^{\prime} ; \omega\right)=\frac{(2 \pi)^{18}}{4 \pi^{4} \Omega^{2}} \sum_{k_{1}} \frac{\Gamma_{B}\left(-k,-k_{1} ; \omega\right) \Gamma_{A}\left(k_{1}, k^{\prime} ; \omega\right)}{k_{1}^{2} k^{\prime 2}} \\
& \times \exp \left[\mathrm{i} R \cdot\left(k_{1}-k^{\prime}\right)\right] .
\end{aligned}
$$

The zero-point energy associated with the roots of the secular determinant is [9]:

$$
\begin{aligned}
& E(R)=\left[\frac{\hbar}{2}\right] \frac{1}{2 \pi \mathrm{i}} \oint \omega \mathrm{d} \omega \frac{\mathrm{d}}{\mathrm{d} \omega} \ln |\mathcal{I}-\mathcal{S}(\omega)| \\
& =\frac{\hbar}{4 \pi \mathrm{i}} \oint \ln |\mathcal{I}-\mathcal{S}(\omega)| \mathrm{d} \omega=\frac{\hbar}{2 \pi} \int_{0}^{\infty} \ln |\mathcal{I}-\mathcal{S}(\mathrm{i} \xi)| \mathrm{d} \xi .
\end{aligned}
$$

The last form is obtained by distorting the contour so as to go along the imaginary axis in the complex $\omega$-plane, and using the evenness of $\mathcal{S}(\omega)$ as a function of $\omega$. It must be stressed that $E(R)$ above is actually the difference between the zero-point energy of the charge oscillation modes of the coupled system of the two atoms, and that of the charge oscillation modes of the two atoms taken separately. This is because the zeroes of $|\mathcal{I}-\mathcal{S}(\omega)|$ occur at the charge oscillation frequencies of the coupled system, whereas its poles occur at that of each of the atoms in the absence of the other [13]. 
The leading term in Eq. (2.19) is

$E(R)=-\frac{\hbar}{2 \pi} \int_{0}^{\infty} \mathrm{d} \xi \operatorname{Tr}[\mathcal{S}(\mathrm{i} \xi)]$.

Using Eq. (2.18) and converting the k-sums to integrals in Eq. (2.20) we get the expression identical with Eq. (2.7).

It is also apparent that taking $\ln |\mathcal{I}-\mathcal{S}(\omega)|$ as in Eq. (2.19), rather than its leading term $\{-\operatorname{Tr}[\mathcal{S}(\omega)]\}$ as in Eq. $(2.20)$ is tantamount to summing a particular class of terms in perturbation theory to all orders. Thus, Eq. (2.19) will give an improved perturbation theoretic estimate of $\mathrm{L}-\mathrm{vdW}$ interaction compared with Eq. (2.7), or equivalently, with Eq. (2.20).

The problem of evaluation of the effect of the substrate on the interaction between physisorbed atoms or molecules thus boils down to evaluating the charge response functions $\Gamma_{A}$ and $\Gamma_{B}$ in the proximity of the substrate. This is what we proceed to do now.

\section{Response in the proximity of the substrate}

We shall evaluate the matrix $\mathcal{S}$ of Sec. 2 for two interacting adsorbed atoms in the usual point dipole approximation, in which the induced charges $\rho_{\mathrm{I}(A, B)}$ and $p_{B}(\omega)$ are the respective induced dipole moments of $A$ and $B$, the equation equivalent to $(2.14)$ is

$$
p_{B}(\omega)=\wp_{B}(\omega) \cdot \mathcal{G}(2,1 ; \omega) \cdot \wp_{A}(\omega) \cdot \mathcal{G}(1,2 ; \omega) \cdot p_{B}(\omega),
$$

where the dyadic Green function $\mathcal{G}(1,2 ; \omega)$ is defined by

$$
\mathcal{G}(1,2 ; \omega) \equiv \nabla_{1} \nabla_{2} \varphi(1,2 ; \omega)
$$

with $\varphi(1,2 ; \omega)$ being the potenlial at $A$ due to a unit charge at $\boldsymbol{R}$ where $B$ is located, oscillating with frequency $\omega . \wp_{A, B}(\omega)$ are the polarizability tensors of the two atoms. For simplicity we shall take $\wp_{A, B}(\omega)$ as scalars, so that the interaction energy in the form equivalent to $\mathrm{Eq}$. (2.20) becomes

$$
E(R)=-\frac{\hbar}{2 \pi} \int_{0}^{\infty} \mathrm{d} \xi \alpha_{A}(\mathrm{i} \xi) \alpha_{B}(\mathrm{i} \xi) \operatorname{Tr}[\mathcal{G}(1,2 ; \mathrm{i} \xi) \mathcal{G}(2,1 ; \mathrm{i} \xi)] .
$$

In free space in the non-retarded limit

$$
\varphi(1,2 ; \omega)=[1 / R] \Rightarrow \operatorname{Tr}[\mathcal{G}(1,2) \mathcal{G}(2,1)]=\left[6 / R^{6}\right],
$$

which when substituted in Eq. (3.3) gives the well known leading term in L-vdW interaction. We shall now consider a few models of the substrate to see the effect of the substrate on the interaction.

\subsection{The spatially non-dispersive dielectric model}

We take two atoms, with the surface geometry and choice of axes as shown in Fig. 2. Each atom can be on either side of the surface depending on the sign of its $z$-coordinate. The material of the substrate is characterized by a spatially non-dispersive dielectric constant $\varepsilon(\omega)$. In this case, $\varphi(1,2 ; \omega)$ can be obtained by the image method in the form

$$
\varphi(1,2 ; \omega)=\frac{1}{\left|r_{1}-r_{2}\right|}-\left[\frac{\varepsilon(\omega)-1}{\varepsilon(\omega)+1}\right] \frac{1}{\left|r_{1}-\bar{r}_{2}\right|} ; \quad z_{1}>0, z_{2}>0
$$




$$
\begin{aligned}
& =\left[\frac{2}{\varepsilon(\omega)+1}\right] \frac{1}{\left|r_{1}-r_{2}\right|} ; \quad z_{1}>0, z_{2}<0 \\
& =\frac{1}{\varepsilon(\omega)}\left[\frac{1}{\left|r_{1}-r_{2}\right|}+\left\{\frac{\varepsilon(\omega)-1}{\varepsilon(\omega)+1}\right\} \frac{1}{\left|r_{1}-\bar{r}_{2}\right|}\right] ; \quad z_{1}<0, z_{2}<0
\end{aligned}
$$

$\overline{\boldsymbol{r}}_{2}$ is the coordinate of the image of $B$, denoted by $\vec{B}$ - this is illustrated in Fig. 2 for the first case. In the last case $B$ will be to the left of the surface and $\bar{B}$ will be to the right.

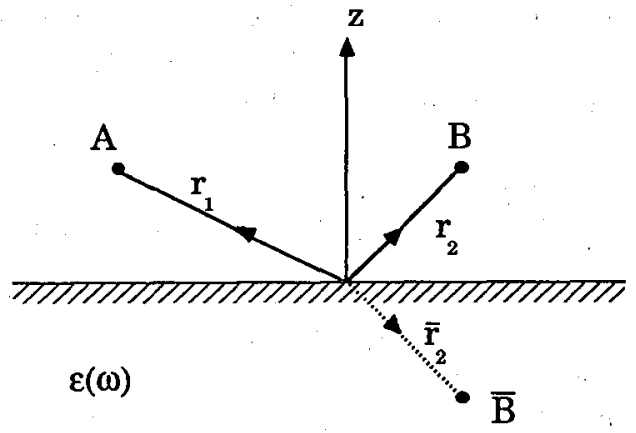

Fig. 2. The molecules outside a spatially non-dispersive dielectric surface. $\bar{B}$ at $\overline{\boldsymbol{r}}_{2}$ is the image of $B$ at $r_{2}$.

In the situation of close proximity to the surface, i.e., when $\left|z_{1}\right|$ and $\left|z_{2}\right|$ are much smaller than $r_{1}$ and $r_{2}$, we get (with $\left|r_{1}-r_{2}\right|=R$ )

$$
\begin{aligned}
& \operatorname{Tr}[\mathcal{G}(1,2 ; \omega) \mathcal{G}(2,1 ; \omega)]=\frac{4\left[\varepsilon^{2}(\omega)+5\right]}{[\varepsilon(\omega)+1]^{2}}\left\{\frac{1}{R^{6}}\right\} ; \quad z_{1}=0^{+}, z_{2}=0^{+} \\
& =\frac{24}{[\varepsilon(\omega)+1]^{2}}\left\{\frac{1}{R^{6}}\right\} ; \quad z_{1}=0^{+}, z_{2}=0^{-} \\
& =\left[\frac{4}{\varepsilon^{2}(\omega)}\right] \frac{\left[5 \varepsilon^{2}(\omega)+1\right]}{[\varepsilon(\omega)+1]^{2}}\left\{\frac{1}{R^{6}}\right\} ; \quad z_{1}=0^{-}, z_{2}=0^{-}
\end{aligned}
$$

For using these results in Eq. (3.3) we need information on both $\alpha_{A, B}(\omega)$ and $\varepsilon(\omega)$ to be able to complete the in tegration over $\xi$. For many substrates information on $\varepsilon(\omega)$ is available from optical data. For $\alpha_{A, B}(\omega)$, a reasonable form which gives good estimates of the strength of $\mathrm{L}-\mathrm{vdW}$ forces, is

$$
\alpha_{A, B}(\omega)=\alpha_{A, B}(0)\left[\frac{\omega_{0(A, B)}^{2}}{\omega_{0(A, B)}^{2}-\omega^{2}}\right],
$$

where $\alpha_{A, B}(0)$ are the static polarizabilities of $A$ and $B$, and $\omega_{0(A, B)}$ are their principal absorption frequencies, the presumption being that all oscillator strengths are 


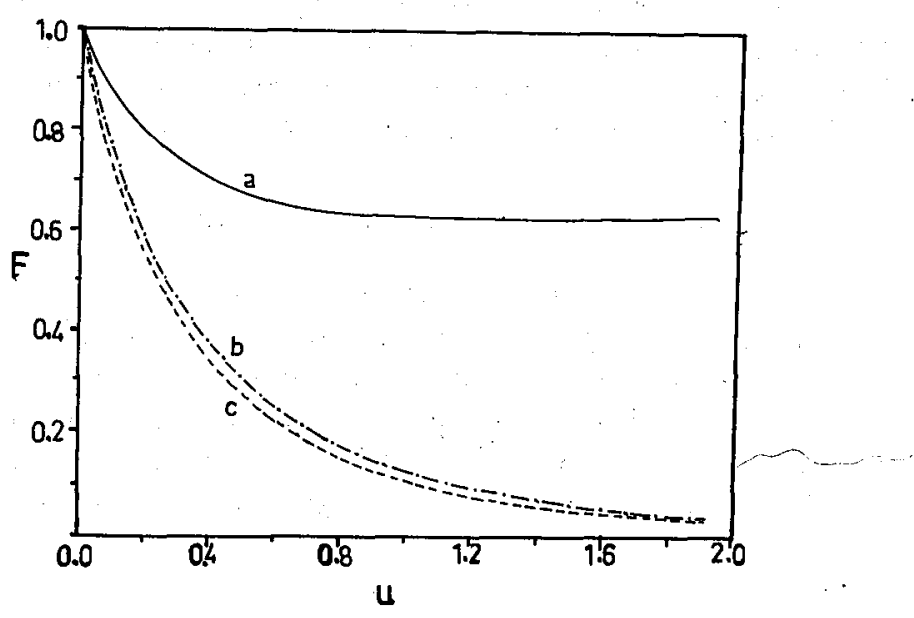

Fig. 3. The ordinate $F$ is the ratio of $\mathrm{L}-\mathrm{vdW}$ interaction of two molecules in free space, to that in their physisorbed state on a metal. The abscissa $u$ is the ratio [ $\left.\omega_{0} / \omega_{s}\right]$. The separation $R$ is arbitrary, but fixed. The labelling of the curves are: (a) the two molecules just outside, (b) one outside and the other just under, and (c) both just under the surface.

concentrated at $\omega_{0(A, B)}$ for the atoms $A$ and $B .\left[\hbar \omega_{0(A, B)}\right]$ is usually of the order of the first ionization potential of $A$ and $B$.

Not many applications of this method, i.e., use of Eqs. (3.3) together with (3.6) and (3.7) have been reported in literature. For the case of a metallic substrate, with the approximation $\varepsilon(\omega)=1-\left[\omega_{\mathrm{P}}^{2} / \omega^{2}\right]=1-\left[2 \omega_{\mathrm{S}}^{2} / \omega^{2}\right]$, where $\omega_{\mathrm{P}}$ is the bulk plasma frequency $\sqrt{\left[4 \pi n_{0} e^{2} / m\right]}$, and $\omega_{\mathrm{S}}\left(\equiv \omega_{\mathrm{P}} / \sqrt{2}\right)$ is the surface plasma frequency, some interesting results ensue [10]. In this case, Eqs. (3.6) reduce to the form

$$
\begin{aligned}
& \operatorname{Tr}[\mathcal{G}(1,2 ; \omega) \mathcal{G}(2,1 ; \omega)] \\
& =\left[\frac{6 \omega^{4}-4 \omega^{2} \omega_{\mathrm{S}}^{2}+4 \omega_{\mathrm{S}}^{4}}{\left(\omega^{2}-\omega_{\mathrm{S}}^{2}\right)^{2}}\right]\left\{\frac{1}{R^{6}}\right\} ; \quad z_{1}=0^{+}, z_{2}=0^{+} \\
& =\left[\frac{6 \omega^{4}}{\left(\omega^{2}-\omega_{\mathrm{S}}^{2}\right)^{2}}\right]\left\{\frac{1}{R^{6}}\right\} ; \quad z_{1}=0^{+}, z_{2}=0^{-} \\
& =\left[\frac{\omega^{4}\left(6 \omega^{4}-20 \omega^{2} \omega_{\mathrm{S}}^{2}+20 \omega_{\mathrm{S}}^{2}\right)}{\left(\omega^{2}-2 \omega_{\mathrm{S}}^{2}\right)^{2}\left(\omega^{2}-\omega_{\mathrm{S}}^{2}\right)^{2}}\right]\left\{\frac{1}{R^{6}}\right\} ; \quad z_{1}=0^{-}, z_{2}=0^{-}
\end{aligned}
$$

Using the form of $\alpha(\omega)$ of Eq. (3.7) for two identical atoms, Eq. (3.3) can be integrated into a closed form for the three locations of interacting atoms, and this form involves the ratio $\left(\omega_{0} / \omega_{\mathrm{S}}\right)$. Figure 3 gives the ratio of the strengths of $L-v d W$ interaction in free space, to that in the adsorbed state on a metallic substrate as a function of $u=\left(\omega_{0} / \omega_{\mathrm{S}}\right)$, at a fixed separation, for the three cases described 
in Eqs. (3.8). The last two cases, corresponding to one or both of the molecules being just below the surface must be interpreted as the situation in which one or both are just imbedded in the electron spill-out region near the metal surface. The weakening of $\mathrm{L}-\mathrm{vdW}$ interaction due to substrate response is obvious.

\subsection{The spatially dispersive dielectric model}

The dielectric response of the substrate is generally more complicated than in the above model, and displays spatial dispersion. The net effect of spatial dispersion is to reduce the weakening of $\mathrm{L}-\mathrm{vdW}$ interaction discussed in Sec. 3.1. The essential features of this process for adsorption on a metallic substrate can be brought out using the hydrodynamic model of the free electrons in a metal [11]. For this model, $\varphi(1,2 ; \omega)$ for $z_{1}>0$ and $z_{2}>0$ in the geometry of Fig. 2 has the

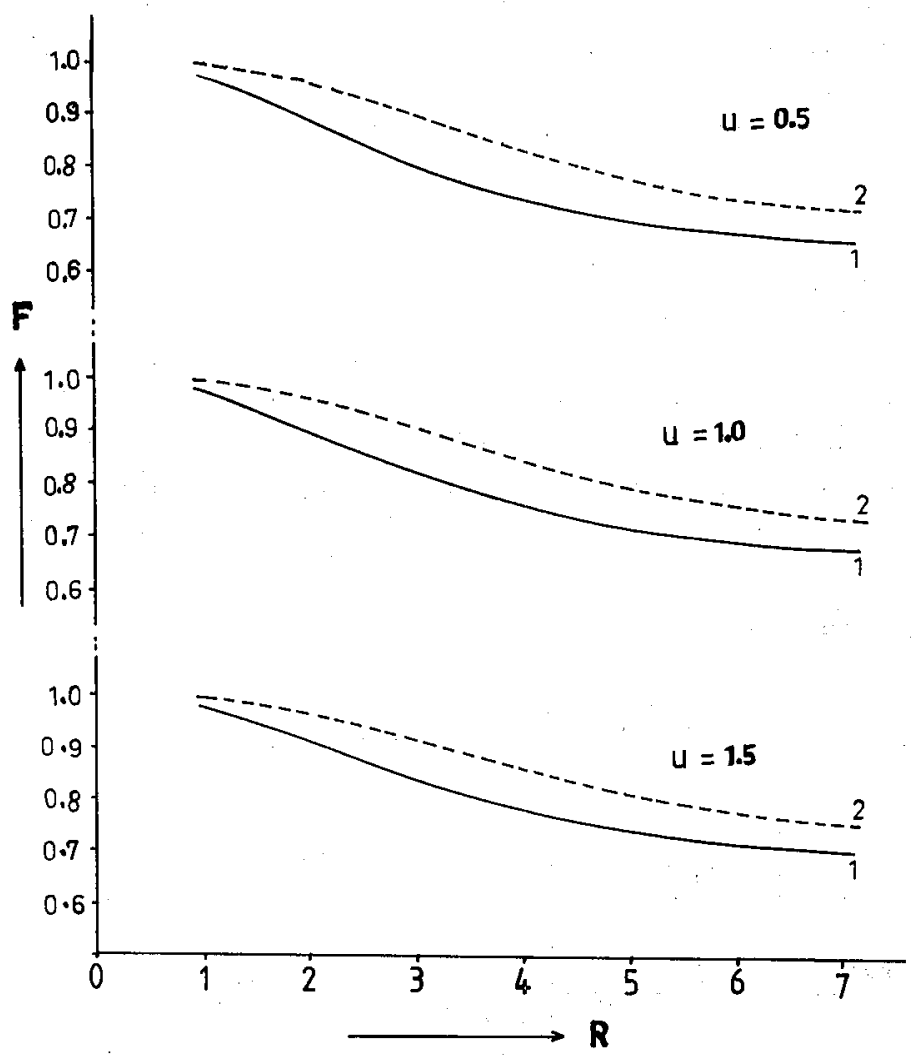

Fig. 4. The ordinate $F$ is the same ratio as in Fig. 3 , for two molecules in the parallel orientation to a spatially dispersive dielectric surface. The abscissa $R$ is the separation distance in unit of $\kappa_{0}^{-1}$. The solid curve (1) is for $z=\kappa_{0}^{-1}$, and the dotted curve (2) is for $z=2 \kappa_{0}^{-1}$. The three sets of curves are for different ratios $u=\left[\omega_{0} / \omega_{\mathrm{P}}\right]$, as indicated. 
form

$$
\begin{aligned}
& \varphi(1,2 ; \omega)=\frac{1}{\left|r_{1}-r_{2}\right|} \\
& -\frac{\kappa_{0}^{2}}{2 \pi} \int \frac{\mathrm{d}^{2} \kappa \exp \left[i \kappa \cdot\left(r_{1(\|)}-r_{2(\|)}\right)-\kappa\left(z_{1}+z_{2}\right)\right]}{\kappa\left[2 \gamma(\gamma+\kappa)-\kappa_{0}^{2}\right]},
\end{aligned}
$$

where $\kappa_{0}^{2} \equiv\left(\omega_{\mathrm{P}}^{2} / \beta^{2}\right)$, with the dispersion parameter $\beta$ given by $\beta^{2}=\left[3 v_{\mathrm{F}}^{2} / 5\right]$, $v_{F}$ being the Fermi velocity; $\gamma^{2}=\kappa^{2}+\left[\omega_{\mathrm{P}}^{2}-\omega^{2}\right] / \beta^{2} . \kappa_{0}^{-1}$ is essentially the Thomas-Fermi screening length in the metal. $r_{1(\|)}$ and $r_{2}(\|)$ are the components of $r_{1}$ and $r_{2}$ parallel to the surface. Figure 4 indicates the same ratio of the interactions as in Sec. 3.1, as a function of the separation distance, for different heights from the surface and different ratios $\left(\omega_{0} / \omega_{P}\right)$. It may be noted that for large separations outside the metal surface, the curves must asymptotically go to the value (2/3), a well known limit for an ideally conducting substrate [12].

\section{Conclusion}

The main object of the analysis presented here is to stress the point that to evaluate the effect of the substrate on the long-range attractive $L-v d W$ part of the interaction of two physisorbed molecules, one needs to evaluate $\varphi(1,2 ; \omega)$ defined after Eq. (3.2). A proper microscopic analysis of the contribution to this from the response of the substrate is essential for quantitatively correct estimates, although the simple models discussed in Secs. 3.1 and 3.2 reveal the main trends.

The behaviour of $\mathrm{L}-\mathrm{vdW}$ interaction for close separations is interesting, since it is radically different from the asymptotic $\left(1 / R^{6}\right)$ behaviour, which of course will diverge for small $R$. Use of the full Eq. (2.20), and not Eq. (3.3) obtained by the point dipole approximation, removes the divergence for $R \rightarrow 0$. Although a computation using Eq. (2.20) has not been made for any system, some phenomenologically pursuasive estimates have confirmed this removal of divergence for $R \rightarrow 0$ [13]. It must be stressed that a proper treatment for small $R$ must use exchange-corrected perturbation theory $[7,8]$.

\section{References}

[1] L.W. Bruch, Surf. Sci. 125, 194 (1983).

[2] N.H. March, in: Interaction of Atoms and Molecules with Solid Surfaces, Eds. V. Bortolani, N.H. March, M.P. Tosi, Plenum Press, New York 1990, p. 1.

[3] T. Takaishi, Prog. Surf. Sci. 6, 45 (1975).

[4] K. Joyce, P.J. Grout, N.H. March, Surf. Sci. Lett. 181, L141 (1987).

[5] K. Kern, G. Gomsa, in: Chemistry and Physics of Solid Surfaces VII, Eds. R. Vanselow, R. Howe, Springer-Verlag, Berlin 1988, p. 65.

[6] F. London, Z. Phys. 63, 245 (1930).

[7] J.O. Hirschfelder, Chem. Phys. Lett. 1, 325 (1967); 1, 363 (1967).

[8] A.A. Rumyantsev, Sov. Phys. JETP 38, 459 (1974). 
[9] A.A. Maradudin, E.W. Montroll, G.H. Weiss, I.P. Ipatova, in: Theory of Lattice Dynamics in the Harmonic Approximation, Academic Press, New York 1971, Ch. VIII.

[10] J. Mahanty, Phys. Rev. B 35, 4113 (1987).

[11] J. Mahanty, B.V. Paranjape, Surf. Sci. 202, 335 (1988).

[12] H. Margenau, N.R. Kestner, in: Theory of Intermolecular Forces, Pergamon Press, Oxford 1971, p. 300 .

[13] J. Mahanty, B.W. Ninham, in: Dispersion Forces, Academic Press, London 1976. 